\title{
Efeitos da dieta hipocalórica sobre a função endotelial em adultos obesos
}

\author{
Effects of caloric restriction diet on endothelial function in obese adults
}

Fabiana de Faria Ghetti ${ }^{1}$, Mateus Camaroti Laterza ${ }^{1}$

1Unidade de Investigação Cardiovascular e Fisiologia do Exercício do Hospital Universitário e Faculdade de Educação Física e Desportos da Universidade Federal de Juiz de Fora (FAEFID/UFJF) - Juiz de Fora (MG), Brasil.

DOI: http://dx.doi.org/10.7322/abcshs.v39i1.248

\section{RESUMO}

A obesidade é uma doença inflamatória crônica caracterizada pelo acúmulo excessivo de gordura no tecido adiposo. Componentes fisiopatológicos decorrentes do excesso de gordura corporal, como alterações no balanço de adipocinas, resistência insulínica e aumento de mediadores inflamatórios, promovem disfunção endotelial, e, consequentemente, maior risco de morbidade e mortalidade de origem cardiovascular. Por outro lado, a adoção de dieta hipocalórica tem sido recomendada como medida não farmacológica para o tratamento da obesidade. Portanto, o objetivo desse artigo foi o de realizar uma revisão de literatura sobre os efeitos da dieta hipocalórica na função endotelial em adultos com obesidade. Foram analisados 26 artigos com os descritores dieta redutora, restrição calórica e perda de peso, combinados com os termos vasodilatação e endotélio, publicados nas bases de dados eletrônicas Medline e Scielo. Foi possível observar que a dieta hipocalórica, quando associada à perda de peso corporal, melhora os parâmetros metabólicos, inflamatórios, hemodinâmicos e neurovasculares, os quais promovem melhora da função endotelial em indivíduos obesos. Entretanto, para que esses benefícios sejam obtidos, a dieta deve ser individualizada, balanceada e com orientação e prescrição de especialistas.

Palavras-chave: obesidade; restrição calórica; endotélio; vasodilatação.

\begin{abstract}
Obesity is a chronic inflammatory disease characterized by excessive accumulation of fat in adipose tissue. Pathophysiological components due to excess of body fat, such as changes in the balance of adipokines, insulin resistance and increased inflammatory mediators, promote endothelial dysfunction and consequently higher risk of morbidity and mortality from cardiovascular causes. On the other hand, energy reduced diet has been recommended as non-pharmacological measure for treatment of obesity. Therefore, the aim of this paper was to realize a literature review on the effects of energy reduced diet on endothelial function in obese adults. Twenty-six papers with descriptions energy reduced diet, calorie restriction and weight loss, combined with the terms vasodilatation and endothelium, published in Medline and Scielo electronic databases were evaluated. It was noted that energy reduced diet, when it results in weight loss, changes metabolic, inflammatory, hemodynamic and neurovascular parameters, with restoration of normal endothelial function in obese subjects. However, to achieve those benefits, the energy reduced diet should be individualized, balanced and with guidance and prescription of experts.
\end{abstract}

Keywords: obesity; caloric restriction; endothelium; vasodilation. 


\section{INTRODUÇÃO}

A obesidade é uma doença crônica caracterizada pelo acúmulo excessivo de gordura no tecido adiposo, provocado por desequilíbrio nutricional associado ou não a distúrbios genéticos ou endocrinometabólicos ${ }^{1}$. Nos Estados Unidos, a prevalência da obesidade é de 33,8\% em homens e de 35,5\% em mulheres ${ }^{2}$. Dados nacionais, divulgados pelo Instituto Brasileiro de Geografia e Estatística (IBGE), revelam que a prevalência de obesidade, em aproximadamente três décadas, aumentou em mais de quatro vezes para homens $(2,8$ para $12,4 \%)$ e em mais de duas vezes para mulheres (de 8,0 para $16,9 \%)^{3}$.

$\mathrm{O}$ excesso de gordura corporal, principalmente na região abdominal, é considerado fator de risco independente para o desenvolvimento de doença arterial coronariana ${ }^{4}$. Um dos possíveis mecanismos que explicam a maior morbidade de origem cardiovascular é a piora da função endotelial ${ }^{5}$. Realmente, foi observado que indivíduos que apresentavam disfunção endotelial tinham risco 3,5 vezes maior de sofrer evento cardíaco em relação aos que apresentavam função endotelial normal ${ }^{6}$. Existem claras evidências de que indivíduos com obesidade possuem perda da função endotelial normal ${ }^{7,8}$, fato que justifica, pelo menos em parte, a maior probabilidade de infarto agudo do miocárdio e morte súbita nessa população?.

Por outro lado, a adoção de dieta hipocalórica tem sido recomendada como medida não farmacológica para o tratamento da obesidade ${ }^{1}$. Em estudo com indivíduos obesos, somente três meses de dieta hipocalórica foram suficientes para reduzir em mais de $5 \%$ o peso corporal ${ }^{10}$. Além disso, ensaios clínicos têm demonstrado que a redução do peso corporal, por meio de dieta hipocalórica, vem acompanhada de melhoras nos componentes fisiopatológicos presentes na obesidade ${ }^{10-13}$. Desta forma, o objetivo desta revisão de literatura foi abordar os efeitos da dieta hipocalórica na função endotelial em adultos com obesidade.

\section{MÉTODO}

A busca bibliográfica consistiu na pesquisa de artigos publicados até o ano de 2012, nas seguintes bases de dados eletrônicas: National Library of Medicine (Medline) e Scientific Electronic Library Online (SciELO). Utilizaram-se os seguintes descritores de saúde para a busca: dieta redutora, restrição calórica e perda de peso, combinados com os termos vasodilatação e endotélio. Idioma e período de publicação não foram restringidos. Todos os ensaios clínicos randomizados e não randomizados que utilizaram para o tratamento da obesidade em adultos apenas dieta hipocalórica para um dos grupos de estudo, tendo como desfecho os efeitos da perda de peso sobre marcadores da função endotelial, tais como: metabólicos, inflamatórios, hemodinâmico e/ou neurovascular. Os estudos que associaram a restrição calórica com cirurgia bariátrica, medicação ou exercício físico, sem comparação com grupo que realizou apenas dieta, foram excluídos desta revisão.

\section{RESULTADOS}

\section{Descrição dos estudos selecionados}

A busca eletrônica retornou 47 ensaios clínicos que avaliaram os efeitos da dieta hipocalórica sobre a função endotelial de pessoas com obesidade. Desses 47 artigos, 28 foram excluídos por associarem dieta hipocalórica com cirurgia bariátrica, medicação ou exercício físico, sem terem um grupo que realizou apenas dieta com a finalidade de comparação. Dos 19 artigos incluídos, dez eram randomizados.

Excluindo os pacientes que não seguiram o protocolo experimental, os 19 artigos incluídos nesta revisão totalizam 620 pacientes. Entre os estudos analisados, a ingestão calórica variou de 580 a $2.000 \mathrm{kcal}$ por dia, por período de intervenção de 4 a 52 semanas. Em relação à composição das dietas hipocalóricas, os carboidratos forneceram 4 a $60 \%$ das calorias diárias, os lipídeos 7 a $75 \%$ e as proteínas 15 a 50\%. A idade dos pacientes variou entre 18 e 69 anos, e o tamanho da amostra variou entre sete e 70 participantes. Quanto ao gênero dos pacientes, Maeda et al. ${ }^{10} \mathrm{e}$ Johnstone et al. ${ }^{13}$ incluíram apenas o gênero masculino e Capel et al. ${ }^{14}$, Mavri et al..$^{15}$ e Morel et al. ${ }^{12}$, apenas o feminino, enquanto os demais estudos incluíram ambos os gêneros.

Apenas Pierce et al. ${ }^{16}$ compararam grupo de obesos que realizou dieta hipocalórica com grupo de obesos que não foi submetido à intervenção (controle). Já Borges et al. ${ }^{17}$ compararam grupo de obesos submetidos à dieta hipocalórica com grupo que, além de realizar a dieta, consumiu medicação para perda de peso. Tonacio et al. ${ }^{8}$, Straznicky et al. ${ }^{18}$ e Wycherley et al. ${ }^{19}$ compararam grupo de pacientes que seguiram dieta hipocalórica com um grupo que associou dieta com exercício físico. Os outros estudos compararam os resultados de um mesmo grupo de pacientes obesos antes a após intervenção.

\section{Função endotelial e obesidade}

O endotélio saudável possui propriedades anti-inflamatórias e antitrombóticas, sendo esse processo mediado pela síntese e liberação de substâncias vasoativas como o óxido nítrico $(\mathrm{ON})$, produzido a partir da ação da enzima óxido nítrico sintase endotelial (eNOS) sobre o substrato L-arginina ${ }^{20}$. Além de ser o mais potente vasodilatador endógeno, o ON também reduz a permeabilidade vascular, a adesão de plaquetas e monócitos, a oxidação e a inflamação tecidual, a ativação de fatores trombogênicos, a proliferação de células lisas musculares, assim como a expressão de citocinas pró-aterogênicas e pró-inflamatórias ${ }^{21}$.

Na obesidade, a função do endotélio encontra-se prejudicada, pois a biodisponibilidade do ON está comprometida por diversos mecanismos relacionados ao excesso de gordura corporal, incluindo a intolerância à glicose, resistência insulínica, desregulação metabólica, síntese de adipocitocinas, estresse oxidativo e inflamação sistêmica ${ }^{22}$.

Embora a disfunção endotelial seja um forte preditor de eventos cardiovasculares, a restauração da homeostase 
arterial reduz o risco de morbidade e mortalidade de origem vascular ${ }^{23}$. Nesse sentido, as evidências atuais têm demonstrado que uma das principais estratégias para restaurar a função endotelial na obesidade é a redução do peso corporal por meio de dieta hipocalórica. Acredita-se que a restrição calórica seja capaz de modificar parâmetros metabólicos ${ }^{24,25}$, inflamatórios ${ }^{13,17}$, hemodinâmicos ${ }^{11,24,26}$ e neurovasculares ${ }^{18}$, os quais são considerados importantes marcadores da função vascular.

\section{Efeitos da dieta hipocalórica sobre a função endotelial em indivíduos obesos}

Estudos clínicos demonstram que a dieta hipocalórica, quando resulta em perda de peso corporal, está associada com melhora na função endotelial de indivíduos obesos ${ }^{11,22,24,27}$. Por exemplo, Varady et al. ${ }^{24}$ observaram que em indivíduos obesos a dilatação mediada por fluxo, ou seja, a vasodilatação dependente do endotélio, aumentou em $32 \%$ após seis semanas de dieta hipocalórica, com perda de $5 \%$, em média, do peso corporal. Mas, esses benefícios podem ser dependentes das características da dieta e do percentual de perda de peso. No estudo de Raitakari et al. ${ }^{11}$, dieta de muito baixa caloria, composta por $7 \%$ de lipídeos, aumentou em $60 \%$ a dilatação mediada por fluxo em obesos, que reduziram, ao final da intervenção, cerca de $11 \%$ do peso corporal.

Embora, no estudo anterior, o aumento significativo no fluxo sanguíneo foi obtido com moderada perda de peso ${ }^{11}$, as evidências atuais consideram que o percentual de redução do peso corporal igual ou superior a $5 \%$ é suficiente para se alcançar os benefícios sobre a função endotelial ${ }^{13,18,24,27,28}$. Porém, a perda de peso corporal, quando obtida por meio de dieta hipocalórica com muito baixo teor de carboidratos ( $4 \%$ das calorias) e elevado teor de lipídeos (61\% das calorias) e proteínas (35\% das calorias), parece não melhorar a função endotelial de indivíduos obesos ou até mesmo prejudicar a dilatação dependente do endotélio ${ }^{29}$.

Os mecanismos que associam a dieta hipocalórica com a recuperação da função endotelial ainda não estão esclarecidos. Entretanto algumas evidências sugerem que as adipocinas, como a adiponectina, leptina e resistina, exercem papel de destaque ${ }^{15,23,24}$. A adiponectina é um hormônio sintetizado na célula adiposa que estimula a síntese de ON pela enzima eNOS endotelial ${ }^{23}$. Ao contrário, a resistina e a leptina são hormônios pró-aterogênicos, também derivados dos adipócitos, que causam disfunção endotelial por aumentar o estresse oxidativo ${ }^{30}$. Parece que a dieta hipocalórica aumenta a produção de adiponectina e reduz a liberação de leptina e resistina ${ }^{11,14,16}$. Em estudo realizado por Varady et al..$^{24}$ foi observado que a restrição de $25 \%$ do requerimento energético diário promoveu aumento de $16 \%$ nos níveis circulantes de adiponectina e redução de 48 e de $26 \%$ nos níveis de leptina e resistina, respectivamente. Além disso, essa melhora nos parâmetros fisiológicos do adipócito foi estatisticamente associada com a melhora da dilatação da artéria braquial após hiperemia reativa ${ }^{24}$.

Em experimento com modelo animal, identificou-se que a adiponectina melhora a vasodilatação por vias sinalizadas pela insulina $^{31}$. Nesse estudo, a restrição calórica aumentou significativamente a concentração plasmática de adiponectina e reduziu a insulinemia, sendo que esses efeitos associaram-se à elevação dos níveis de eNOS fosforilada e ao aumento da síntese de $\mathrm{ON}^{31}$. Dessa forma, os autores sugerem que a insulina pode ser um regulador determinante dos efeitos benéficos da restrição calórica, uma vez que esse hormônio, em níveis adequados no plasma sanguíneo, atua na fosforilação e ativação da NOS $^{31}$. Ensaios clínicos também sugerem que, após dieta hipocalórica, há melhora significativa da resistência insulínica em pessoas com obesidade ${ }^{19,25,32,33}$. Independente da composição da dieta, o índice que avalia a homeostase de resistência à insulina diminuiu em até $57 \%$ em indivíduos obesos, ao final da intervenção com restrição de calorias ${ }^{25}$.

Adicionalmente, outros ensaios demonstram que os níveis glicêmicos dessas pessoas podem melhorar após perda de peso e controle da insulinemia ${ }^{17,19}$. Embora a redução de até $25 \%$ da glicemia foi obtida com dieta hipocalórica ${ }^{19}$, percentual menor de queda da concentração da glicose plasmática já se correlaciona com melhora da dilatação da artéria braquial frente a estímulo fisiológico ${ }^{11}$. Uma das possíveis explicações para essa relação positiva é a diminuição do estresse oxidativo gerado pela auto-oxidação da glicose e a redução da formação de produtos de glicosilação avançada, como a hemoglobina glicada $(\mathrm{HbAlc})^{16}$. De fato, Whycherley et al. ${ }^{19}$ mostraram que o consumo de 1.200 calorias diárias por obesos, após 12 semanas, resultou em menores níveis plasmáticos de HbA1c.

Existem evidências de que para se alcançar redução significativa do estresse oxidativo na obesidade, a dieta hipocalórica deve promover perda de peso corporal igual ou superior a $5 \%$ do peso inicial ${ }^{19,27}$. A partir desse percentual, nota-se que marcadores da biodisponibilidade de ON na célula endotelial, como o nitrito e nitrato plasmáticos, apresentam o dobro da concentração em relação aos valores basais. Além disso, quanto maior a concentração de nitrito e nitrato, maior a vasodilatação dependente do endotélio ${ }^{27}$.

Outro possível mecanismo capaz de explicar a redução do estresse oxidativo e, portanto, a melhora da função endotelial em indivíduos obesos, é o controle da inflamação crônica subclínica. Os marcadores de inflamação crônica subclínica, como o fator de necrose tumoral (TNF- $\alpha)^{13}$, a interleucina- 6 (IL-6) e proteína-C reativa $(\mathrm{PCR})^{34}$, estão diretamente relacionadas com obesidade ${ }^{34}$. Acredita-se que os marcadores inflamatórios, além de causar estresse oxidativo ${ }^{35}$, podem aumentar a resistência insulínica ${ }^{34} \mathrm{e}$ a produção de proteínas que ativam a adesão de leucócitos ao endotélio vascular ${ }^{21}$. Por outro lado, a dieta hipocalórica é capaz de reverter esse processo inflamatório subclínico. Há evidências de que a restrição 
calórica, acompanhada de perda de peso superior a 5\%, diminui, principalmente, a PCR e o TNF- $\alpha$, cujos níveis circulantes podem ser, em média, $60 \%$ menores em relação aos níveis basais $^{15,17}$. Uma possível explicação para esse resultado seria a melhora no balanço de adipocinas e insulinemia, que ocorre com a perda ponderal ${ }^{11,34}$.

Sugere-se, além disso, que a modulação da inflamação crônica subclínica pela adiponectina possa ser responsável pela redução de moléculas de adesão celular, incluindo a molécula-1 de adesão intracelular (ICAM-1), a molécula-1 de adesão celular vascular (VCAM-1) e a E-seletina, geralmente associadas com a patogênese da aterosclerose e preditores de eventos clínicos $^{35,36}$. Esses marcadores endoteliais estão elevados na obesidade e são consistentemente reduzidos com a perda de peso corporal, após dieta hipocalórica ${ }^{12,28,37,38}$. Similarmente, fatores fibrinolíticos derivados do endotélio e micropartículas derivadas de plaquetas e leucócitos também estão envolvidos na disfunção endotelial de obesos, e seus níveis circulantes podem ser controlados com a restrição de calorias da dieta ${ }^{12,38}$.

$\mathrm{Na}$ obesidade, ainda, foi constatado que a restrição calórica reduz a síntese de endotelina-1 (ET-1), um potente peptídeo vasoconstritor envolvido na disfunção endotelial. Em estudo realizado por Maeda et al. ${ }^{10} \mathrm{com}$ homens obesos, a perda de peso promovida por dieta hipocalórica reduziu significativamente, em três meses, os níveis de ET-1. Além disso, quanto maior a perda de peso corporal, maior a redução desse vasoconstritor no plasma sanguíneo $(\mathrm{r}=0,87 ; \mathrm{p}<0,05)^{10}$. Portanto, a redução da ET-1 pela perda de peso corporal pode melhorar a disfunção endotelial causada pela obesidade, uma vez que a ET-1 está envolvida na regulação do tônus vascular e progressão da aterosclerose.

O efeito benéfico da restrição de calorias sobre o tônus vascular também pode ser explicado pelo controle da atividade do sistema nervoso simpático que ocorre com a perda de peso corporal $^{8}$. De fato, estudo clínico demonstrou que perder mais de $5 \%$ do peso inicial, após dieta hipocalórica, reduz significativamente a hiperatividade simpática ${ }^{18}$. Provavelmente, esse resultado é mediado pelo balanço das adipocinas, já que os níveis plasmáticos de leptina reduziram em pelo menos 50\%, em apenas três meses de intervenção ${ }^{18}$.

A melhora da função endotelial associada com a perda ponderal ainda pode ser mediada pelo efeito positivo da dieta hipocalórica sobre os níveis séricos de lipoproteínas. No estudo realizado por Pierce et al. ${ }^{16}$, a dieta hipocalórica promoveu redução significativa da lipoproteína de baixa densidade oxidada (LDL-ox), e este efeito pode ser uma das explicações para a melhora da vasodilatação da artéria braquial, evidenciada no grupo de obesos submetidos à restrição calórica. Segundo esse autor, o controle da concentração sérica de LDL-ox parece aumentar a biodisponibilidade de ON nas células endoteliais, tornando-se importante marcador do estresse oxidativo ${ }^{16}$.
Entre os lipídeos plasmáticos, os triglicerídeos são os que mais respondem positivamente à dieta hipocalórica ${ }^{13}$. Embora dietas com baixo teor de carboidratos promovam reduções significativas dos triglicerídeos em curto período de tempo ${ }^{13,30}$, em seis meses, o consumo de dietas hipocalóricas com teor moderado desse macronutriente é capaz de reduzir a trigliceridemia em $60 \%$ dos valores basais ${ }^{25}$. Portanto, independente da composição da dieta, a restrição calórica contribui para o controle da hipertrigliceridemia na obesidade, e consequentemente, para a restauração da função endotelial normal, uma vez que níveis elevados de triglicerídeos estão relacionados à redução das lipoproteínas de alta densidade, resistência insulínica e pressão arterial elevada, que contribuem para o aumento do risco de aterosclerose ${ }^{39}$. Todos esses mecanismos do efeito da dieta hipocalórica sobre a função endotelial de adultos obesos estão esquematizados na Figura 1.

\section{CONSIDERAÇÕES FINAIS}

Assim, com base nesses dados da literatura, verificamos que a dieta hipocalórica acompanhada de redução de peso corporal melhora os marcadores da função endotelial em pessoas com obesidade (Tabela 1).

De acordo com US National Institutes of Health ${ }^{40}$, a dieta planejada individualmente para criar déficit de 500 a $1.000 \mathrm{kcal}$ por dia, com no mínimo de 1.000 a $1.200 \mathrm{kcal} / \mathrm{d}$ para as mulheres e $1.200 \mathrm{a} 1.400 \mathrm{kcal} / \mathrm{d}$ para os homens, deve ser parte integrante de qualquer programa de perda de peso que objetive diminuição de 0,5 a $1,0 \mathrm{~kg}$ por semana. As dietas balanceadas são compostas de 20 a $30 \%$ de gorduras, 55 a $60 \%$ de carboidratos e 15 a $20 \%$ de proteínas. Além disso, o tratamento dietético é mais bem-sucedido quando aliado a aumento no gasto energético e ao programa de modificação comportamental ${ }^{1}$.

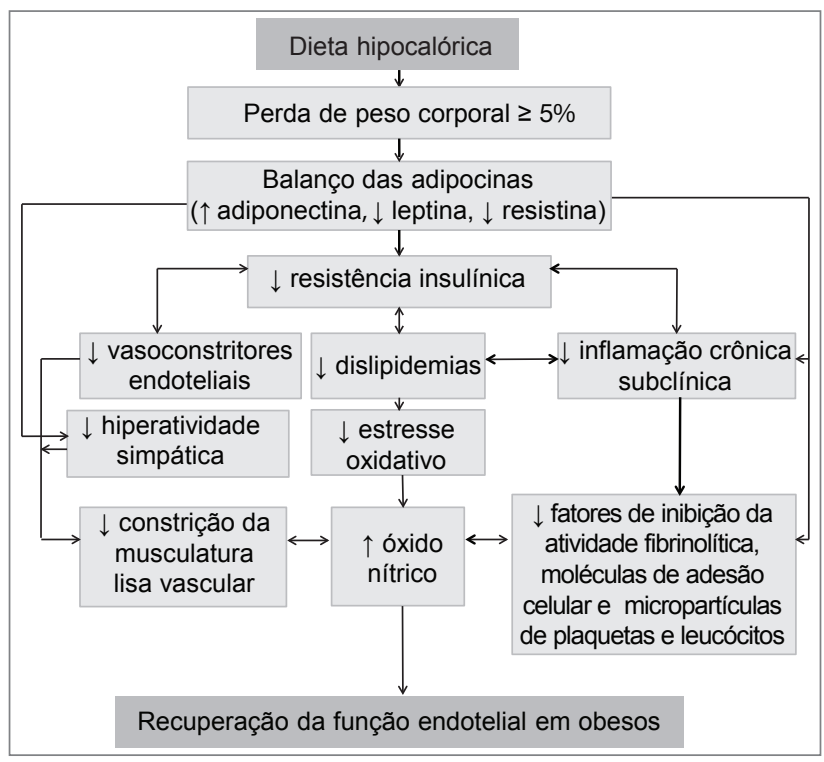

Figura 1: Mecanismos que associam a dieta hipocalórica com a melhora da função endotelial na obesidade 
Tabela 1: Efeitos da dieta hipocalórica nos marcadores da função endotelial em adultos obesos

\begin{tabular}{|c|c|c|}
\hline Autor (ano) & Intervenção & Efeitos $(p<0,05)$ \\
\hline $\begin{array}{l}\text { Maeda et al. }{ }^{10} \\
(2010)\end{array}$ & $3 \mathrm{~m}(\mathrm{n}=7) ; 1.680 \mathrm{kcal} / \mathrm{d}$. PP: $13 \%$. & $\downarrow 18 \%$ ET- 1. \\
\hline Raitakari et al. ${ }^{11}(2004)$ & 6 sem $(n=67) ; 580 \mathrm{kcal} / \mathrm{d}$. PP: $11 \%$. & $\begin{array}{c}\uparrow 60 \% \text { DMF, } \uparrow 19 \% \text { adiponectina, } \\
\downarrow 42 \% \text { PCR, } \downarrow 35 \% \text { LDL-ox, } \downarrow 43 \% \text { insulinemia, } \downarrow \text { \& } \% \text { glicemia. }\end{array}$ \\
\hline $\begin{array}{l}\text { Morel et al. }{ }^{12} \\
(2011)\end{array}$ & $\begin{array}{c}2 \mathrm{~m}(\mathrm{n}=24) ; 600 \mathrm{kcal} / \mathrm{d}(1 \mathrm{~m}) \text { e } 1.200 \mathrm{kcal} / \mathrm{d}(2 \mathrm{~m}) . \\
\text { PP: } 10 \% .\end{array}$ & $\downarrow 43 \%$ MPs de plaquetas, $\downarrow 28 \%$ MPs de leucócitos, $\downarrow 32 \%$ leptina, $\downarrow 38 \%$ PAl- 1 . \\
\hline $\begin{array}{l}\text { Johnstone et al. }{ }^{13} \\
\text { (2011) }\end{array}$ & 4 sem $(n=12) ; 2.000 \mathrm{kcal} / \mathrm{d} ;$ LC vs MC. PP: 6 vs $4 \%$. & $\begin{array}{c}\text { LC vs MC: } \\
\downarrow 19 \text { vs } \downarrow 37 \% \text { IL-10, } \downarrow 58 \text { vs } \downarrow 24 \% \text { TNF- } \alpha, \downarrow 45 \text { vs } \downarrow 37 \% \text { HOMA-IR, } \downarrow 61 \text { vs } \\
\downarrow 49 \% \text { Leptina, } \downarrow 12 \text { vs } \downarrow 15 \% \text { LDL, } \downarrow 43 \text { vs } \downarrow 39 \% \text { TG. }\end{array}$ \\
\hline $\begin{array}{l}\text { Capel et al. }{ }^{14} \\
(2009)\end{array}$ & $\begin{array}{c}2 \mathrm{~m}(\mathrm{n}=22) ; 800 \mathrm{kcal} / \mathrm{d}\left(1^{\circ} \mathrm{m}\right) \text { e restrição de } \\
600 \mathrm{kcal} / \mathrm{d} \\
\left(2^{\circ} \mathrm{m}\right) . \mathrm{PP}: 11 \%\end{array}$ & $\downarrow 65 \%$ insulinemia, $\downarrow 27 \%$ TG, $\downarrow 11 \%$ CT, $\downarrow 26 \%$ PCR, $\downarrow 42 \%$ leptina. \\
\hline $\begin{array}{l}\text { Mavri et al. }{ }^{15} \\
(2011)\end{array}$ & $5 \mathrm{~m}(\mathrm{n}=22) ; 1.200 \mathrm{kcal} / \mathrm{d} . \mathrm{PP}: 17 \%$. & 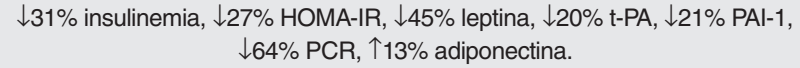 \\
\hline $\begin{array}{l}\text { Pierce et al. }{ }^{16} \\
(2008)\end{array}$ & 12 sem $(n=26) ; 1.200 \mathrm{kcal} / \mathrm{d} . \mathrm{PP}: 10 \%$. & $\begin{array}{c}\text { Dieta vs Controle: } \\
\downarrow 225 \% \text { vs s/a insulinemia, } \downarrow 228 \% \text { vs s/a HOMA-IR, } \downarrow 17 \% \text { vs s/a LDL-ox, } \\
\downarrow 40 \% \text { vs s/a leptina, } \uparrow 30 \% \text { vs s/a DMF. }\end{array}$ \\
\hline $\begin{array}{l}\text { Borges et al. }{ }^{17} \\
(2007)\end{array}$ & $\begin{array}{l}16 \text { sem }(n=24) \text {; dieta hipocalórica vs dieta } \\
\text { hipocalórica + orlistat. PP: } 8 \% \text { ambos os grupos. }\end{array}$ & $\begin{array}{c}\text { Dieta vs Dieta }+ \text { orlistat: } \\
\downarrow 5 \text { vs } \downarrow 14 \% \text { glicemia, } \downarrow 56 \text { vs } \downarrow 60 \% \text { TNF- } \alpha, \downarrow 16 \text { vs } \downarrow 20 \% \text { leptina. }\end{array}$ \\
\hline $\begin{array}{l}\text { Straznicky et al. }{ }^{18} \\
(2011)\end{array}$ & $\begin{array}{l}12 \text { sem }(n=18) ; \text { restrição de } 600 \mathrm{kcal} / \mathrm{d} \text {. } \\
\text { PP: } 7 \% \text {. }\end{array}$ & $\downarrow$ ANSM em 25 disparos por 100 batimentos cardíacos, $\downarrow 52,8 \%$ leptina. \\
\hline $\begin{array}{l}\text { Wycherley et al. }{ }^{19} \\
(2008)\end{array}$ & $\begin{array}{c}12 \text { sem ( } n=29) ; 1.200 \mathrm{kcal} / \mathrm{d} \text { vs Dieta + Exercício. } \\
\text { PP: } 9 \% \text { ambos os grupos. }\end{array}$ & $\begin{array}{c}\text { Dieta vs Dieta }+ \text { Exercício: } \\
\downarrow 225,5 \text { vs } \downarrow 23,5 \% \text { glicemia, } \downarrow 20,4 \text { vs } \downarrow 15,5 \% \text { HbA1c, } \downarrow 39,4 \text { vs } \downarrow 59,1 \% \\
\text { HOMA-IR, } \downarrow 27 \text { vs } \downarrow 26 \% \text { MDA, } \uparrow 86,7 \text { vs } \downarrow 69,8 \% \text { nitrito/nitrato urinário. }\end{array}$ \\
\hline $\begin{array}{l}\text { Varady et al. }{ }^{24} \\
\text { (2011) }\end{array}$ & $\begin{array}{c}6 \mathrm{sem}(\mathrm{n}=10) ; \mathrm{LF}(1.967 \mathrm{kcal} / \mathrm{d}) \text { : vs HF }(1.868 \mathrm{kcal} / \mathrm{d}) . \\
\text { PP: } 5 \text { vs } 7 \% .\end{array}$ & $\begin{array}{c}\text { LF vs HF: } \\
\uparrow 32 \text { vs } \downarrow 19 \% \text { DMF, } \uparrow 16 \% \text { vs s/a adiponectina, } \downarrow 26 \% \text { vs s/a resistina, } \\
\downarrow 48 \text { vs } \downarrow 28 \% \text { leptina. }\end{array}$ \\
\hline $\begin{array}{l}\text { Brinkworth et al. }{ }^{33} \\
\text { (2009) }\end{array}$ & $\begin{array}{c}52 \mathrm{sem}(\mathrm{n}=69) ; 1.463 \mathrm{kcal}(()) \text { a } 1.707 \mathrm{kcal} / \mathrm{d}\left({ }^{\top}\right) ; \text { LC: } \\
\text { vs LF. PP: } 14 \% \text { ambos os grupos. }\end{array}$ & $\begin{array}{l}\text { LF vs LC: } \\
\downarrow 34 \text { vs } \downarrow 43 \% \text { insulinemia, } \downarrow 37 \text { vs } \downarrow 41 \% \text { HOMA-IR, } \downarrow 50 \text { vs } \downarrow 48 \% \text { PCR. }\end{array}$ \\
\hline $\begin{array}{l}\text { Russo et al. }{ }^{25} \\
(2010)\end{array}$ & $6 \mathrm{~m}(\mathrm{n}=20) ; 1.200$ a $1.800 \mathrm{kcal} / \mathrm{d}$. PP: $14 \%$. & $\downarrow 53 \%$ insulina, $\downarrow 57 \%$ HOMA-IR, $\downarrow 13 \%$ CT, $\downarrow 60 \%$ TG, $\uparrow 27 \%$ HDL. \\
\hline $\begin{array}{l}\text { Volek et al. }{ }^{26} \\
(2009)\end{array}$ & 12 sem ( $n=40) ; 1.500$ kcal/d; LC: vs LF. PP: 10 vs 5\%. & $\begin{array}{c}\text { LC vs LF: } \\
\uparrow 27,5 \text { vs } \downarrow 34,2 \% \text { DMF, } \downarrow 47 \text { vs } \downarrow 15 \% \text { TG, } \downarrow 51 \text { vs } \downarrow 2 \% \text { insulinemia. }\end{array}$ \\
\hline $\begin{array}{l}\text { Angelico et al. }{ }^{27} \\
(2012)\end{array}$ & 6 m (n=53); Restrição de 600 kcal/d. PP: 7\%. & $\begin{array}{c}\text { Grupo } \geq 5 \% \text { PP vs }<5 \% \text { PP: } \\
\uparrow 24,7 \% \text { vs s/a DMF, } \uparrow 116,8 \% \text { vs s/a NOX, } \uparrow 125,5 \% \text { vs s/a adiponectina, } \\
\downarrow 22,2 \% \text { vs s/a NOX } 2 .\end{array}$ \\
\hline $\begin{array}{l}\text { Davis et al. }{ }^{28} \\
(2011)\end{array}$ & 6 m (n=27); LC vs LF. PP: $6 \%$ ambos os grupos. & $\begin{array}{c}\text { LC vs LF: } \\
\text { s/a vs } \downarrow 25 \% \text { PCR, } \downarrow 15 \% \text { vs s/a ICAM- } 1, \downarrow 12 \% \text { vs s/a E-seletina. }\end{array}$ \\
\hline $\begin{array}{l}\text { Schulte et al. }{ }^{32} \\
(2012)\end{array}$ & $12 \operatorname{sem}(n=23) ; 800 \mathrm{kcal} / \mathrm{d}$. PP: 15\%. & $\downarrow 50 \%$ HOMA-IR, $\downarrow 63 \%$ LAR, $\downarrow 56 \%$ leptina. \\
\hline $\begin{array}{l}\text { Keogh et al. }{ }^{38} \\
(2008)\end{array}$ & 8 sem $(n=70) ; 1.463 \mathrm{kcal} / \mathrm{d}$. LC: vs HC. PP: 8 vs $6 \%$. & 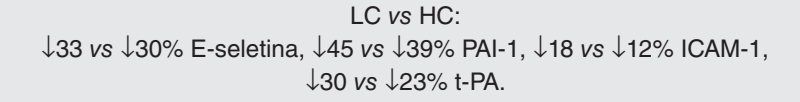 \\
\hline $\begin{array}{l}\text { McLaughlin et al. }{ }^{37} \\
(2006)\end{array}$ & $\begin{array}{c}16 \operatorname{sem}(n=57) \text {; restrição de } 750 \mathrm{kcal} / \mathrm{d}: \mathrm{HC} \text { vs } \mathrm{MC} . \\
\text { PP: } 7 \text { vs } 6 \%\end{array}$ & $\begin{array}{c}\text { HC vs MC: } \\
\text { s/a vs } \downarrow 22 \% \text { E-seletina, s/a vs } \downarrow 28 \% \text { TG, } \downarrow 12 \% \text { vs } \downarrow 32 \% \text { insulinemia. }\end{array}$ \\
\hline
\end{tabular}

ANSM: atividade nervosa simpática neuromuscular; CT: colesterol total; HC: dieta rica em carboidrato; HF: dieta rica em gordura; LC: dieta pobre em carboidrato; LF: dieta pobre em gordura; MC: dieta moderada em carboidrato; DMF: dilatação mediada por fluxo; ET-1: endotelina-1; HbA1c: hemoglobina glicada; HDL: lipoproteína de alta densidade; HOMA-IR: modelo de avaliação da homeostase de resistência à insulina; ICAM-1: molécula de adesão intercelular-1; IL-10: interleucina 10; LAR: razão leptina/adiponectina; LDL-ox: lipoproteína de baixa densidade oxidada; m:meses; MDA: malondialdeído; MPs: micropartículas; NOX: nitrito e nitrato; NOX2: núcleo catalítico da NADPH oxidase; PAI-1: inibidor do ativador do plasminogênio 1; PCR: proteína-C reativa; PP: perda de peso; s/a: sem alteração significativa; sem: semanas; TG: triglicérides; TNF- $\alpha$ : fator de necrose tumoral; tPA: ativador de plasminogênio tipo tecidual; VCAM-1: molécula de adesão celular vascular-1 


\section{REFERÊNCIAS}

1. Associação Brasileira para o Estudo da Obesidade e da Síndrome Metabólica. Diretrizes brasileiras de obesidade 2009/2010. 3. ed. Itapevi: AC Farmacêutica; 2009. p. 85.

2. Flegal KM, Carroll MD, Ogden CL, Curtin LR. Prevalence and trends in obesity among US adults, 1999-2008. JAMA. 2010;303(3):235-41.

http://dx.doi.org/10.1001/jama.2009.2014

3. Instituto Brasileiro de Geografia e Estatística. Pesquisa de Orçamentos Familiares 2008-2009: antropometria e estado nutricional de crianças, adolescentes e adultos no Brasil. Rio de Janeiro: IBGE; 2010.

4. Coutinho T, Goel K, Corrêa de Sá D, Carter RE, Hodge DO, Kragelund $\mathrm{C}$, et al. Combining body mass index with measures of central obesity in the assessment of mortality in subjects with coronary disease: role of "normal weight central obesity". J Am Coll Cardiol. 2013;61(5):553-60.

http://dx.doi.org/10.1016/j.jacc.2012.10.035

5. Shankar SS, Steinberg HO. Weight loss and vascular function: the good and the unknown. Hypertension. 2008;52(1):57-8. http://dx.doi.org/10.1161/HYPERTENSIONAHA.108.112441

6. Lerman A, Zeiher AM. Endothelial function: cardiac events. Circulation. 2005;111(3):363-8 http://dx.doi.org/10.1161/01.CIR.0000153339.27064.14

7. Van Guilder GP, Stauffer BL, Greiner JJ, Desouza CA. Impaired endothelium-dependent vasodilation in overweight and obese adult humans is not limited to muscarinic receptor agonists. Am J Physiol Heart Circ Physiol. 2008;294(4):1685-92.

http://dx.doi.org/10.1152/ajpheart.01281.2007

8. Tonacio AC, Trombetta IC, Rondon MU, Batalha LT, Kuniyoshi $\mathrm{FH}$, Laterza MC, et al. Effects of diet and exercise training on neurovascular control during mental stress in obese women. Braz J Med Biol Res. 2006;39(1):53-62. http://dx.doi.org/10.1590/S0100-879X2006000100006

9. Whitlock G, Lewington S, Sherliker P, Clarke R, Emberson J, Halsey $\mathrm{J}$, et al. Body-mass index and cause-specific mortality in 900.000 adults: collaborative analyses of 57 prospective studies. Lancet. 2009;373(9669):1083-96. http://dx.doi.org/10.1016/S0140-6736(09)60318-4

10. Maeda S, Jesmin S, Iemitsu M, Otsuki T, Matsuo T, Ohkawara K, et al. Weight loss reduces plasma endothelin-1 concentration in obese men. Exp Biol Med. 2006;231(6):1044-47.

11. Raitakari $M$, Ilvonen $T$, Ahotupa $M$, Lehtimäki $T$, Harmoinen $A$, Suominen $\mathrm{P}$, et al. Weight reduction with very-low-caloric diet and endothelial function in overweight adults: role of plasma glucose. Arterioscler Thromb Vasc Biol. 2004;24(1):124-8. http://dx.doi.org/10.1161/01.ATV.0000109749.11042.7c

12. Morel O, Luca F, Grunebaum L, Jesel L, Meyer N, Desprez $\mathrm{D}$, et al. Short-term very low-calorie diet in obese females improves the haemostatic balance through the reduction of leptin levels, PAI-1 concentrations and a diminished release of platelet and leukocyte-derived microparticles. Int $\mathrm{J}$ Obes (Lond). 2011;35(12):1479-86.

http://dx.doi.org/10.1038/ijo.2011.19

13. Johnstone AM, Lobley GE, Horgan GW, Bremner DM, Fyfe CL, Morrice PC, et al. Effects of a high-protein, low-carbohydrate v. high-protein, moderate-carbohydrate weight-loss diet on antioxidant status, endothelial markers and plasma indices of the cardiometabolic profile. Br J Nutr. 2011;106(2):282-91. http://dx.doi.org/10.1017/S0007114511000092

14. Capel F, Klimcáková E, Viguerie N, Roussel B, Vítková M, Kováciková M, et al. Macrophages and adipocytes in human obesity: adipose tissue gene expression and insulin sensitivity during calorie restriction and weight stabilization. Diabetes. 2009;58(7):1558-67.

http://dx.doi.org/10.2337/db09-0033

15. Mavri A, Poredoš P, Suran D, Gaborit B, Juhan-Vague I, Poredoš P. Effect of diet-induced weight loss on endothelial dysfunction: early improvement after the first week of dieting. Heart Vessels. 2011;26(1):31-8.

http://dx.doi.org/10.1007/s00380-010-0016-1

16. Pierce GL, Beske SD, Lawson BR, Southall KL, Benay FJ, Donato AJ, et al. Weight loss alone improves conduit and resistance artery endothelial function in young and older overweight/obese adults. Hypertension. 2008:52(1):72-9.

http://dx.doi.org/10.1161/HYPERTENSIONAHA.108.111427

17. Borges RL, Ribeiro-Filho FF, Carvalho KMB, Zanella MT. Impacto da perda de peso nas adipocitocinas, na proteína C-Reativa e na sensibilidade à insulina em mulheres hipertensas com Oobesidade central. Arq Bras Cardiol. 2007;89(6):371-5 http://dx.doi.org/10.1590/S0066-782X2007001800010

18. Straznicky NE, Grima MT, Eikelis N, Nestel PJ, Dawood T, Schlaich MP, et al. The effects of weight loss versus weight loss maintenance on sympathetic nervous system activity and metabolic syndrome components. J Clin Endocrinol Metab. 2011;96(3):E503-8. http://dx.doi.org/10.1210/jc.2010-2204

19. Wycherley TP, Brinkworth GD, Noakes M, Buckley JD, Clifton PM Effect of caloric restriction with and without exercise training on oxidative stress and endothelial function in obese subjects with type 2 diabetes. Diabetes Obes Metab. 2008;10(11):1062-73. http://dx.doi.org/10.1111/j.1463-1326.2008.00863.x

20. de la Sierra A, Larrousse M. Endothelial dysfunction is associated with increased levels of biomarkers in essential hypertension. $J$ Hum Hypertens. 2010;24(6):373-9. http://dx.doi.org/10.1038/jhh.2009.91

21. Cabral MD, Teixeira PFS, Leite SP, Vaisman M. Marcadores de função endotelial no hipotireoidismo. Arq Bras Endocrinol Metab. 2009;53(3):303-09 http://dx.doi.org/10.1590/S0004-27302009000300002

22. Bigornia SJ, Mott MM, Hess DT, Apovian CM, McDonnell ME, Duess MA, et al. Long-term successful weight loss improves vascular endothelial function in severely obese individuals. Obesity. 2010;18(4):754-9.

http://dx.doi.org/10.1038/oby.2009.482

23. Hirata Y, Nagata D, Suzuki E, Nishimatsu H, Suzuki J, Nagai $R$. Diagnosis and treatment of endothelial dysfunction in cardiovascular disease. Int Heart J. 2010:51(1):1-6. http://dx.doi.org/10.1536/ihj.51.1

24. Varady KA, Bhutani S, Klempel MC, Phillips SA. Improvements in vascular health by a low-fat diet, but not a high-fat diet, are mediated by changes in adipocyte biology. Nutr J. 2011;10:8 http://dx.doi.org/10.1186/1475-2891-10-8

25. Russo I, Traversa M, Bonomo K, De Salve A, Mattiello L, Del Mese $P$, et al. In central obesity, weight loss restores platelet sensitivity to nitric oxide and prostacyclin. Obesity . 2010;18(4):788-97. http://dx.doi.org/10.1038/oby.2009.302

26. Volek JS, Ballard KD, Silvestre R, Judelson DA, Quann EE, Forsythe $\mathrm{CE}$, et al. Effects of dietary carbohydrate restriction versus low-fat diet on flow-mediated dilation. Metabolism. 2009;58(12):1769-77. http://dx.doi.org/10.1016/j.metabol.2009.06.005

27. Angelico F, Loffredo L, Pignatelli P, Augelletti T, Carnevale $\mathrm{R}$, Pacella $A$, et al. Weight loss is associated with improved endothelial dysfunction via NOX2-generated oxidative stress 
down-regulation in patients with the metabolic syndrome. Intern Emerg Med. 2012;7(3):219-27.

http://dx.doi.org/10.1007/s11739-011-0591-x

28. Davis NJ, Crandall JP, Gajavelli S, Berman JW, Tomuta N, WylieRosett $\mathrm{J}$, et al. Differential effects of low-carbohydrate and lowfat diets on inflammation and endothelial function in diabetes. J Diabetes Complications. 2011;25(6):371-6. http://dx.doi.org/10.1016/j.jdiacomp.2011.08.001

29. Wycherley TP, Brinkworth GD, Keogh JB, Noakes M, Buckley JD, Clifton PM. Long-term effects of weight loss with a very low carbohydrate and low fat diet on vascular function in overweight and obese patients. J Intern Med. 2010;267(5):452-61. http://dx.doi.org/10.1111/j.1365-2796.2009.02174.x

30. Krysiak R, Handzlik-Orlik G, Okopien B. The role of adipokines in connective tissue diseases. Eur J Nutr. 2012;51(5):513-28. http://dx.doi.org/10.1007/s00394-012-0370-0

31. Cerqueira FM, Brandizzi LI, Cunha FM, Laurindo FR, Kowaltowski AJ. Serum from calorie-restricted rats activates vascular cell enos through enhanced insulin signaling mediated by adiponectin. PLoS One. 2012;7(2):e31155. http://dx.doi.org/10.1371/journal.pone.0031155

32. Schulte DM, Müller N, Neumann K, Oberhaüser F, Faust $\mathrm{M}$, Güdelhöfer $\mathrm{H}$, et al. Pro-inflammatory wnt5a and antiinflammatory sfrp5 are differentially regulated by nutritional factors in obese human subjects. PLoS One. 2012;7(2): e32437. http://dx.doi.org/10.1371/journal.pone.0032437

33. Brinkworth GD, Noakes M, Buckley JD, Keogh JB, Clifton PM. Longterm effects of a very-low-carbohydrate weight loss diet compared with an isocaloric low-fat diet after 12 mo. Am J Clin Nutr. 2009;90(1):23-32. http://dx.doi.org/10.3945/ajcn.2008.27326
34. Kopp HP, Krzyzanowska K, Möhlig M, Spranger J, Pfeiffer AFH, Schernthaner $\mathrm{G}$. Effects of marked weight loss on plasma levels of adiponectin, markers of chronic subclinical inflammation and insulin resistance in morbidly obese women. Int $\mathrm{J}$ Obes. 2005;29(7):766-71.

http://dx.doi.org/10.1038/sj.ijo.0802983

35. De Keulenaer GW, Alexander RW, Ushio-Fukai M, Ishizaka N, Griendling KK. Tumour necrosis factor alpha activates a p22phoxbased NADH oxidase in vascular smooth muscle. Biochem J. 1998;329(Pt 3):653-7.

36. Ouchi N, Higuchi A, Ohashi K, Oshima Y, Gokce N, Shibata R, et al. Sfrp5 is an anti-inflammatory adipokine that modulates metabolic dysfunction in obesity. Science. 2010; 329(5990):454-7. http://dx.doi.org/10.1126/science. 1188280

37. McLaughlin T, Carter S, Lamendola C, Abbasi F, Yee G, Schaaf P, et al. Effects of moderate variations in macronutrient composition on weight loss and reduction in cardiovascular disease risk in obese, insulin-resistant adults. Am J Clin Nutr. 2006;84(4):813-21.

38. Keogh JB, Brinkworth GD, Noakes M, Belobrajdic DP, Buckley JD, Clifton PM. Effects of weight loss from a very-low-carbohydrate diet on endothelial function and markers of cardiovascular disease risk in subjects with abdominal obesity. Am J Clin Nutr. 2008;87(3):567-76.

39. Hennig B, Toborek M, McClain CJ. High-energy diets, fatty acids and endothelial cell function: implications for atherosclerosis. J Am Coll Nutr. 2001;20(2 Suppl):97-105.

http://dx.doi.org/10.1080/07315724.2001.10719021

40. National Heart Lung and Blood Institute [internet]. Clinical guidelines on the identification, evaluation, and treatment of overweight and obesity in adults. Disponível em: <http://www.nhlbi.nih.gov/ guidelines/obesity/ob_home.htm>. Acesso em: 08 ago 2013. 